\title{
Early impact of COVID-19: Observations from an integrated WASH and NTD project in two south-eastern states in India
}

\author{
Shyamala Anand ${ }^{\mathrm{a}}$, Radhika Mamidi ${ }^{\mathrm{b}} \&$ Pritha Biswas $^{\mathrm{c}}$ \\ a Senior Technical Advisor for NTDs, American Leprosy Missions, USA \\ ${ }^{\mathrm{b}}$ State Coordinator-Andhra Pradesh, LEPRA Society, India \\ ${ }^{\mathrm{c}}$ State Coordinator-Odisha, LEPRA Society, India
}

Submitted and accepted 14 August 2020

\begin{abstract}
Summary The COVID-19 pandemic in India has impacted all segments of the population but it has been particularly detrimental to the most vulnerable and has also impacted implementation of health and development projects among these populations. This report describes the early impact (March-August 2020) of the COVID19 pandemic on people affected by leprosy and lymphatic filariasis (LF) and their households in an integrated WASH and NTD project in two south-eastern states in India.
\end{abstract}

Keywords: COVID-19 and leprosy, lockdown and leprosy, lymphatic filariasis

\section{Background}

American Leprosy Missions and LEPRA Society implement an integrated WASH and NTD programme in selected rural villages in NTD endemic districts in Andhra Pradesh (AP) and Odisha states in India.

Every village has key implementers who are trained women volunteers known as Community Resource Persons (CRPs) and key influencers for WASH behaviour change which are the women's self-help groups (SHGs). The programme is ongoing in 37 villages comprising 13,162 households , with a population of 56,346. Agricultural labour is the main source of livelihood. Around $30 \%$ of the working population is involved in seasonal migrant labour within and outside their home states.

This report describes the early impact (March-August 2020) of the COVID-19 pandemic on the project and people affected by leprosy and lymphatic filariasis (LF) and their households living in the project villages. The Government of India imposed a nationwide lockdown on 25 March, 2020 as a preventive measure against the COVID-19 pandemic, which was later extended in phases until 31 May, 2020. It included suspension of public transport services,

Correspondence to: Shyamala Anand, Senior Technical Advisor for NTDs, American Leprosy Missions, USA (e-mail: shyamala@leprosy.org) 
closure of all religious, commercial and educational establishments, offices, and factories and banned public gatherings. Establishments engaged in the supply of essential goods and services were excluded from this lockdown. Millions of migrant workers lost their jobs and started the reverse migration back to their villages on foot. Many were detained in migrant shelters until the phased 'Unlock' which began on 1 June, 2020. This mass human movement aided the spread of COVID-19 from urban to previously relatively unexposed rural areas. ${ }^{1-3}$

\section{Economic and health impact of COVID-19 on people affected by leprosy and lymphatic filariasis}

255 people affected by leprosy (108) and LF (147) from different households live in the 37 project villages in Odisha and AP. 3 of the 255 are children. Of the 255, 40 have Grade 2 leprosy disability and 147 have lymphoedema. 60 men and 58 women are the only income-earning household members and are engaged in daily-wage labour. The other 137 are dependents of the household breadwinner - a spouse, parent or adult child. Since the lockdown began there has been scarcity of regular work. Many have lost their daily wages due to closure of their workplaces and restrictions on travel to work, negatively impacting their household income. During the lockdown, the Gram Panchayats (local government) distributed cooked food and dry rations for two weeks. The government has instituted a cash transfer programme and Public Distribution Supply of dry rations to the poor as an economic response to COVID-19 but the amounts provided by these schemes are insufficient for a household for a month, and it has become difficult to meet basic needs ${ }^{1}$. So, despite the restrictions and the risk of COVID-19, people are going out of their villages on bicycles or bullock carts in search of work.

Limited or no public transport and a nearly five-fold increase in autorickshaw fare has prohibited access to healthcare services. The National Leprosy Program and LEPRA (ILEP partner in AP and Odisha) are ensuring supply of MDT, self-care kits and ulcer-care kits to the Blocks. However, with the health system completely focused on COVID-19 control, stocks at the Block level are not always reaching the Primary Health Centres (PHCs) on time. Frontline health workers (ASHAs) are engaged in COVID-19 work and there has been some delay in delivering MDT to the people. 5 persons are on MDT for leprosy and have been able to continue treatment despite the delays. All 40 people with Grade 2 leprosy disability and 147 people with lower limb lymphoedema have been receiving their essential supply of self-care kits from the nearest PHCs. With restricted PHC outpatient services and government advisories to visit PHCs only in cases of emergencies, out of pocket household health expenditure has increased as people have to buy medicines from local pharmacies for comorbidities like hypertension, diabetes and for general ailments. Fear and stigma associated with COVID-19 have affected health reporting. One PHC in the project area has observed that they have not seen a single case of dengue or malaria this season because people are afraid that if they come with fever, they will be tested for COVID-19.

\section{Impact of COVID-19 on Project implementation}

In response to the COVID-19 situation and government COVID-19 regulations, the project immediately made COVID-19 personal protection mandatory and staff and CRPs were trained on the required aspects. The project rescheduled some activities and modified others. All trainings, meetings and group gatherings have stopped. School-related activities are on hold until schools reopen. Practicing social distancing and wearing masks, CRPs in every village continue to generate awareness on COVID-19 + WASH + NTDs focusing on households and 
individuals rather than groups. They visit door to door and follow up people with leprosy and LF disability for self-care motivation, check on MDT and self-care material supply, identify necessary referrals and facilitate appointments with PHCs for people affected by leprosy and LF needing care.

The first cases of COVID-19 were reported in the AP project area in mid-May and in the Odisha project area in early July. 16 of the 19 project villages in Odisha have been totally sealed since July with no entry of outsiders allowed and in 6 of these villages even internal mobility is restricted. In AP, 13 of the 18 villages are within COVID-19 containment zones ${ }^{4}$. Despite this, only 1 of the 50 CRPs has resigned due to her family's fear of her getting infected.

The government's focus on COVID-19 prevention has positively impacted the project's social and behaviour change communication (SBCC) activities. Promotion of hand hygiene, respiratory hygiene, physical distancing and sanitation is being well received. Public perception of the project's importance has increased along with cooperation and goodwill of the community and local government. CRPs joined government workers in relief material distribution and COVID-19 screening. SHGs in project villages were contracted by the government to tailor 60,000 cloth masks. The project is engaging in COVID-19 awareness in village quarantine centres. In Odisha, the project was asked to take the lead in supervising construction of toilets and handwashing stations in the quarantine centres.

\section{Conclusions}

The COVID-19 pandemic has been particularly detrimental to those dependent on daily labour as their source of income. Many are without reserves for basic essentials. Restrictions on mobility and government focus on COVID-19 have made access to government services including healthcare difficult. Fear of COVID-19 and its stigma have affected health reporting of febrile illnesses. This is true for people affected by leprosy and LF as for others in their communities. ${ }^{5}$ The partnership between the National Leprosy Program and ILEP member organizations on the ground has ensured continuity of treatment and care for people affected by leprosy and $\mathrm{LF}^{6}$. An important lesson concerns how an integrated WASH and NTD project with household level focus and trained and committed volunteers in every village has naturally transitioned to strengthening the COVID-19 response at the grassroots.

\section{Recommendations}

Recommendations are based on identified gaps in the COVID-19 response that equally affect all communities-

(1) Address the psychosocial aspects of COVID-19: Improve government COVID-19 services to build public confidence and dispel fears regarding quarantine and isolation services. Address the stigma associated with COVID-19 to prevent negative consequences.

(2) Increase SBCC for social responsibility to comply with behaviours that prevent transmission.

(3) Ensure essential facilities and supplies to communities in containment zones.

(4) Ensure availability of healthcare in the PHCs for non COVID-19 illnesses.

Further follow-up as the pandemic unfolds and shared experiences from other NTD projects will inform the continued impact of this pandemic on people affected by leprosy and other NTDs. 


\section{References}

1 India under COVID-19 lockdown VOLUME 395, ISSUE 10233,P1315,APRIL 25, 2020 Editorial. https:// www.thelancet.com/journals/lancet/article/PIIS0140-6736(20)30938-7/fulltext.

2 Science Direct Archives of MedicalResearch. Opinion.COVID-19 Sets off MassMigrationinIndia. https://ww w.sciencedirect.com/science/article/pii/S0188440920309401.

3 Ministry of Home Affairs, Government of India Guidelines for Lockdown and Unlock.

4 Ministry of Health \& Family Welfare Government of India Containment Plan Novel Coronavirus Disease 2019 (COVID 19) Version 2 (updated 16.05.2020).

5 Persons Affected by Leprosy and the COVID-19 Global Health Crisis Persons Affected by Leprosy and the COVID-19 Global Health Crisis: GPZL Working Group 2 Consultative Calls Report.

${ }^{6}$ COVID-19: WHO issues interim guidance for implementation of NTD programmes. 